\title{
"A study on Aromatic Amino acid Auxotrophs of Rhizobium leguminosarum biovar trifolii “"
}

\author{
Dr. Raad Hassani Sultan \\ Department of Biology / College of Education \\ University of Mosul
}

\author{
Received \\ 18 / 06 / 2008
}


(RH57, RH114 and RH70), one (RH53) for phenyalanine, two (RH61 and RH84) for tyrosine and one (RH99) for all these amino acids. According to biochemical and intermediates accumulation studies tryptophan auxotrophic mutants could classified into two trpE mutants (RH57 and RH114) and one trp auxotroph (RH70), was tryptophan synthase mutant. Normal symbiotic activity as indicated by mean dry shoot weight, was observed in the tryptophan synthase mutant (RH70). The plants inoculated with aro and trpE mutants fixed nitrogen but significantly less than that of the RH49 inoculated plants, whereas the tyrosine and phenylalanine auxotrophic mutants inoculated clover plants were completely ineffective in nitrogen fixation. The clover plant host seems to provide tryptophan but not tyrosine and phenylalanine to bacteroids in nodules.

\section{Introduction}

Symbiotic interactions between rhizobia and legumes involve a complex interplay of signals culminating in co-ordinated gene expression (1-3). The rhizobial bacteria depend on their host for carbon and energy sources but very little is known about the requirement of other metabolites (4). Numerous symbiotic mutants of rhizobia have been isolated after UV irradiation (5), nitrous acid mutagenesis (6), N-methyl-Ń-nitro-Nnitrosoguanidine (7), acridine orange (8) and transposon (9). Rhizobia require the availability of 20 amino acids for the establishment of effective symbiosis with legumes. Some of these amino acids are synthesized by rhizobium, whereas the plant supplies others (4). The mutants of S. meliloti blocked in the later part of the arginine biosynthetic pathway, in either ornithine transcarbamylase or argininosuccinate synthetase, were effective in nitrogen fixation $(10,11,12)$. Two aspartic acid mutants of $S$. fredii HH303 formed nodules on soybean but these nodules lacked the characteristic pink colour inside and were unable to fix nitrogen. Addition of aspartic acid to the plant growth medium did not restore symbiotic effectiveness to the mutants (13).

Twenty one asparagine auxotrophs of $S$. meliloti 104A14 were isolated using nitrous acid mutagenesis followed by penicillin enrichment. Seventeen auxotrophs formed nodules on alfalfa plants but these nodules were unable to fix nitrogen (12). Hom et al. (14) reported that five auxotrophs of $B$. japonicum USDA110 showed an altered expression of nitrogenase activity in free-living cultures. Three distinct $S$. meliloti loci involved in glutamine biosynthesis $(g \ln A, g \ln I I$ and $g \ln T)$ have been cloned and characterized. None of these loci was found to be essential for symbiotic nitrogen fixation (15). Three glycine-dependent auxotrophic mutants of 


\section{Dr. Raad Hassani Sultan}

S. meliloti 2011 were isolated and these auxotrophs were more effective in nitrogen fixation than the parental strain (16). The histidine auxotrophs of S. meliloti L5-30 induced ineffective nodules (17).

All ilvD mutants of $S$. meliloti induced effective nodules on alfalfa plants (18), indicating that the activity of the dihydroxy acid dehydratase enzyme is not essential for the effective symbiosis of $S$. meliloti with alfalfa plants. All leucine auxotrophs have been reported to induce ineffective nodules on alfalfa plants (18). Two lysine auxotrophs of S. meliloti Rm41 were isolated and the symbiotic properties of these auxotrophs have not been reported (19). It was concluded that the praline gene proC is essential for symbiosis and the mutant does not obtain an exogenous supply of praline in association with soybeans sufficient to satisfy its auxotrophy (20). The present work focuses on the generating of amino acid auxotrophs by nitrous acid and study role of aromatic amino acid pathway in symbiosis.

\section{Materials and Methods}

\section{Bacterial strain and plant cultivar}

Rhizobium leguminosarum bv. trifolii strain was isolated according to Vincent (21). The clover plants were collected from agroclimatic location of Ninavah province-Iraq. Clover seeds were obtained from local market.

\section{Confirmation test of infection of roots of host plant}

To confirm that the isolated strain RH49 is Rhizobium leguminosarum bv. trifolii, a procedure of Vincent (21) was followed by confirmation of infection of roots of clover plants.

\section{Media}

Tryptone Yeast Extract (TY) medium (22). This medium was used for growing, maintenance of rhizobial strains and for testing motility. TY swarm plates contained $0.3 \%(\mathrm{w} / \mathrm{v})$ agar (23).

Mannitol Salt Yeast Extract (MSY) (24). This medium was used for testing succinylated exopolysaccharide, cyclic $\beta-(1 \rightarrow 3)$ cyclic glucans and cellulose fibrils production.

Rhizobial Minimal Medium RMM (25). This medium was used for isolating of rhizobial auxotrophs, intermediate feeding studies, intermediate accumulation studies and utilization of different sugars and dicarboxylic acids.

Nitrogen free (NF) Plant Medium (26). This medium was used for authentication and symbiotic response of aromatic amino acid auxotrophs.

\section{Maintenance of rhizobial auxotrophic strains}


Pure auxotrphic strains were streaked on slants of TY solid medium. After a growth period of $24-48$ hours at $28 \pm 2{ }^{\circ} \mathrm{C}$, slants were stored at $4{ }^{\circ} \mathrm{C}$ in a refrigerator. Subculturing of rhizobial strains was done each two months.

\section{Supplements to media}

Amino acid/ amino acid intermediates

Stock solutions of aromatic amino acids, i.e. tryptophan, tryptophan intermediates (anthranilic acid, indole and shikmic acid), tyrosine and phenylalanine were made in distilled water and sterilized by passing them through $0.45 \mu \mathrm{m}$ membrane filters and stored at $4{ }^{\circ} \mathrm{C}$. The final concentrations used for aromatic amino acid and tryptophan intermediates were $50 \mu \mathrm{m} / \mathrm{ml}$. Required volumes from the stock solution were added to the autoclaved media before plating.

Sugars and dicarboxylic acids

Sugars (glucose, maltose, raffinose, and sucrose), mannitol and dicarboxylic acids (malic acid, aspartic acid and sodium succinate) were added at a final concentration of $2 \mathrm{~g} / \mathrm{l}$ to the RMM without glucose as a sole carbon source before autoclaving.

Dyes

Calcofour white for testing production of succinolyted exopolysaccharide was added to the MSY medium at the rate of $0.02 \%$ (w/v), while Congo red for testing cellulose fibrils production was added to the same medium at $0.1 \mathrm{mg} / \mathrm{ml}$ final concentration. Each of these dyes was added to the medium before autoclaving (23).

\section{Nitrous acid mutagenesis and penicillin enrichment}

The procedure of nitrous acid mutagenesis of $R$. leguminosarum bv. trifolii strain RH49 was carried out according to Kerpolla and Kahn (12).

Nitrous acid $\left(\mathrm{HNO}_{2}\right)$ preparation

Nitrous acid was prepared in the laboratory just before start of mutagenesis by dissolving $0.035 \mathrm{gm}$ of sodium nitrite $\left(\mathrm{NaNO}_{2}\right)$ in $5 \mathrm{ml}$ of $0.1 \mathrm{M}$-sodium acetate buffer ( $\mathrm{pH} 4.6)$ (12).

Sodium acetate buffer ( $p H 4.6)$ preparation

To prepare $0.1 \mathrm{M}$-sodium acetate buffer $(\mathrm{pH} 4.6)$, in a volumetric flask $100 \mathrm{ml}$, mix $25.5 \mathrm{ml}$ of acetic acid $(0.2 \mathrm{M})$ with $24.5 \mathrm{ml}$ sodium acetate $(0.2 \mathrm{M})$. Complete the volume to $100 \mathrm{ml}$ with distilled water and sterilized with autoclave.

Nitrous acid mutagenesis procedure

A $10 \mathrm{ml}$ culture was grown overnight in TY broth medium. The cells were washed in $0.1 \mathrm{M}$-sodium acetate buffer $(\mathrm{pH} 4.6)$ and resuspended in $1 \mathrm{ml}$ of freshly prepared nitrous acid. Samples were withdrawn after 10, 15 
and $20 \mathrm{~min}$. after the start of mutagenesis, washed to remove the nitrous acid, and then grown in TY broth medium for $8 \mathrm{hr}$ allowed reverse mutagenesis to segregate. The cells were washed in $0.85 \% \mathrm{NaCl}$ and grown for $6 \mathrm{hr}$ in Rhizobial Minimal Medium to deplete nutrient pools. Penicillin was added to final concentration of $300 \mu \mathrm{g} / \mathrm{ml}$ and the culture was shaken for $2 \mathrm{hr}$ to kill $\mathrm{trp}^{+}$or $p h e^{+}$rhizobia that were still growing in the Minimal Medium and to keep on trp rhizobial cells. After the cells had been washed twice in $0.85 \% \mathrm{NaCl}$, serial dilutions were plated on TY agar medium. The survival rate was determined by titrating the culture before and after mutagenesis.

\section{Isolation of aromatic amino acid auxotrophs}

Colonies from mutagenenized cultures were replica-picked onto Rhizobial Minimal and TY complete medium and were classified for aromatic amino acid auxotrophy by testing for growth on RMM supplemented with aromatic amino acids or tryptophan intermediates (27), and then incubated for $3-5$ days at $28 \pm 2{ }^{\circ} \mathrm{C}$.

Biochemical characterization of aromatic amino acid auxotrophs

Intermediate feeding studies

The tryptophan auxotrophs were streaked on RMM medium supplemented with anthranilic acid, indole, or tryptophan and aro mutants on RMM medium supplemented with shikmic acid. The growth patterns of auxotrophs were recorded after an incubation period of 3-5 days at $28 \pm 2{ }^{\circ} \mathrm{C}$. Intermediate accumulation studies and cross feeding assays

Tryptophan auxotrophs were inoculated in minimally supplemented (with $2 \mu \mathrm{g} / \mathrm{ml}$ tryptophan) RMM liquid medium and incubated for 3 days. The supernatant obtained after centrifugation of the culture was assayed for the presence or absence of anthranilic acid and indole using $p$-dimethyl aminobenzaldehyde reagent as described by Snell and Snell (28), and indole glycerol phosphate using ferric chloride reagent according to the method of Yanofsky and Smith (29). Cross feeding assays were done by streaking different combinations of trp auxotrophs close to each other on minimally supplemented (with $2 \mu \mathrm{g} / \mathrm{ml}$ tryptophan) RMM solid medium. The growth of the strains was followed over a period of 3-5 days at $28 \pm 2{ }^{\circ} \mathrm{C}$.

\section{Plant inoculation studies}

Clover seeds were sterilized as described by Wang et al. (30) and transferred onto nitrogen free agar slants in $20 \times 2.5 \mathrm{~cm}$ tubes. Two 2-days old seedlings in each tube were inoculated with $10^{8}$ cells (suspended in sterile distilled water) of a particular rhizobial strain, i.e. RH49, RH70, RH99, RH57, RH61 and RH53. The growth conditions for the plants were 
2000 lux light, a photoperiod of $16 \mathrm{hr}$, a dark period of $8 \mathrm{hr}$ and $25{ }^{\circ} \mathrm{C}$ temperature. The morphological features of plants were recorded six weeks after inoculation (21). Determining the dry plant shoot weight was taken as evidence of nitrogen fixation according to Wang et al. (30). The plant tops were collected and dried in an oven at $65^{\circ} \mathrm{C}$ for $72 \mathrm{hr}$ and then weighted. Reisolation of bacteria from nodules was done to confirm the nodule occupancy by a particular strain. If nodules on a plants were found to be inhabited by prototrophic revertant cells the data on the plant were discarded.

\section{Statistical analysis}

The data which obtained from the plant inoculation studies were statically analysed as described by Somasegaran and Hoben (31).

\section{Results and discussion}

\section{Isolation of R. leguminosarum bv. trifolii strain}

One local strain (RH49) of $R$. leguminosarum bv. trifolii was isolated from root nodules of clover plants.

\section{Confirmation of infection of roots of clover host plant}

Confirmation test of infection of isolated strain (RH49) of roots of clover plants showed that this strain has the ability of inducing nodules on the roots of the mentioned plants, this result means a specific isolated strain of Rhizobium leguminosarum bv. trifolii that inter a relationship with clover plants.

\section{Isolation of aromatic amino acid auxotrophs}

$R$. leguminosarum bv. trifolii strain was mutaginized by with nitrous acid and treated with penicillin to enrich for auxotrophs. Among cultures with $0.1 \%$ survival rates, a $2.4 \%$ frequency of auxotrophic mutants was observed when rhizobial cell treated with nitrous acid for 15 min. Out of 2750 colonies tested, 66 grew on TY agar medium but did not grown on rhizobial minimal medium. Out of these mutants, 7 aromatic amino acid auxotrophic mutants were obtained. Out of these mutants three (RH57, RH114 and RH70) were auxotrophic for tryptophan, one (RH53) for phenylalanine, two (RH61 and RH84) for tyrosine and one (RH99) for all these three amino acids.

\section{Location of biochemical block in tryptophan}

Out of three tryptophan (trp) auxotrophs, two (RH57 and RH114) grew on anthranilic acid supplementation and did not accumulate any tryptophan intermediate, these two were hence $\operatorname{trpE}$ mutants. The RH70 trp 
mutant, was tryptophan synthase mutant since it grew only on tryptophan supplementation and accumulated anthranilic acid and indole glycerol phosphate. The two trpE mutants (RH57 and RH114) were cross-fed by tryptophan synthase mutant (RH70). The mutant RH99 required the supplementation of all three aromatic amino acids for their growth on minimal medium. The mutation in this mutant was possibly in the common aromatic pathway and hence these were called aro mutant. This mutant did not grow on shikmic acid supplementation. The possible location of biochemical block in each auxotroph has been given in Fig. 1.

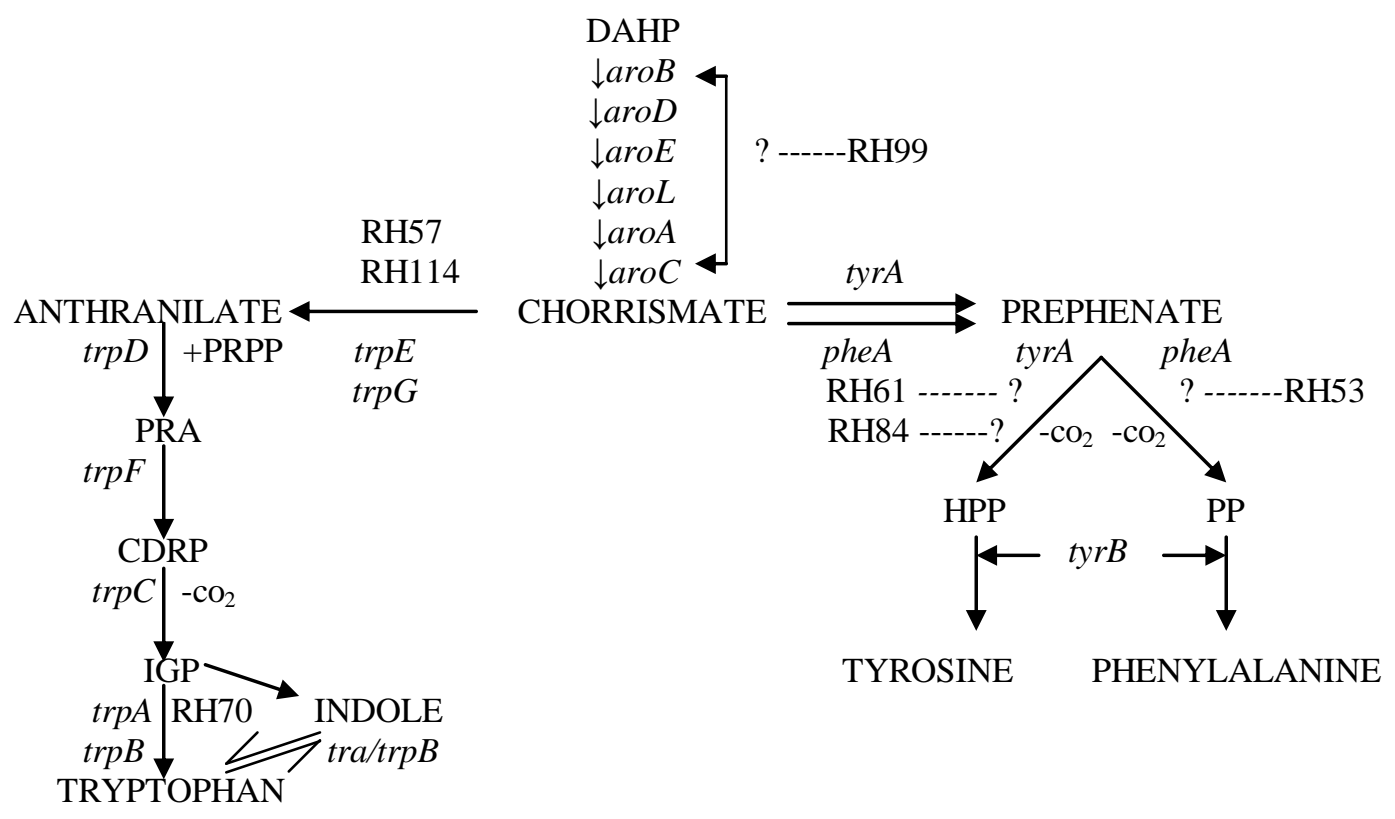

Fig. 1- Pathways of synthesis of aromatic amino acids showing positions of mutations in the auxotrophs obtained. Abbreviations: DAHP, 3 hydroxy-D-arabino-heptulosonate-7-phosphate; PRA, Phosphoribosyl anthranilate; CDRP, 1-(o-carboxyphenylamino)-1-deoxyribulose-5-phosphate; IGP, Indole glycerol phosphate; HPP, 4-Hydroxy phenylpyruvate; PP. Phenylpyruvate [after Moat and Foster (32)].

\section{Pleiotropic effects of auxotrophy}

\section{Production of cell surface molecules}

All the aromatic amino acid auxotrophs and the parental strain RH49 took up the dye congo red, showed motility in swarm medium and fluoresced in presence of calcofluor white under ultra violet light, indicating the normal production of cellulose fibrils, $\beta-(1 \rightarrow 3)$ cyclic glucans and exopolysaccharides, respectively. These results showed that the cell surface molecules in auxotrophs were a like those of the parental strain.

Utilization of sugars and dicarboxilic acids 
All aromatic amino acid auxotrophs showed growth similar to that of the parental strain RH49 on minimal medium supplemented with the respective auxotrophic requirement. No change in the growth behavior of the auxotrophs was detected when glucose in RMM was replaced by any one of the other sugars (glucose, maltose, raffinose and sucrose) and mannitol or dicarboxylic acids (malic acid, aspartic acid and sodium succinate) as carbon source.

The production of cell surface molecules and utilization of sugars and mannitol or dicarboxilic acids indicated that the nitrous acid mutagenesis has no pleiotropic effects on aromatic amino acid auxotrophs.

\section{Symbiotic properties}

The nodules induced by the tryptophan synthase mutant (RH70) were pink and cylindrical like those induced by the parental strain RH49. The symbiotic effectiveness (as indicated by mean dry shoot weight of the inoculated plants) of this strain was exactly like that of the parental strain. On the other hand, the plants inoculated with the aro (RH99) and trpE (RH57) mutants became chlorotic six weeks after inoculation. The mean dry shoot weight of the plants inoculated with an aro or trpE mutants was significantly less than that of the RH49 inoculated plants but significantly more than that of the uninoculated plants. The nodules induced by the aro and $\operatorname{trp} E$ mutants were white and cylindrical. The plants inoculated with tyrosine and phenylalanine mutants did not differ significantly from the uninoculated plants with respect to dry shoot weight. The nodules induced by this mutant were irregular and white in colour (Table 1 ).

Table 1: Nodule characteristics and mean dry shoot weights of plants inoculated with aromatic amino acid auxotrophs of $R$. leguminosarum bv. trifolii RH41 strain

\begin{tabular}{|c|c|c|c|}
\hline \multirow{2}{*}{ Strain } & \multicolumn{2}{|c|}{ Nodule characteristics } & \multirow{2}{*}{$\begin{array}{c}\text { Mean dry shoot } \\
\text { weight }^{\mathbf{a}}(\mathbf{m g})\end{array}$} \\
\cline { 2 - 3 } & Colour & Shape & $5.8 \pm 1.1$ \\
\hline Control & ------ & ------ & $31.3 \pm 1.4$ \\
\hline RH49 & Pink & Cylindrical & $29.5 \pm 0.9$ \\
\hline RH70 & -do- & - do- & $* 11.4 \pm 1.7$ \\
\hline RH99 & White & - do- & $* 12.1 \pm 1.5$ \\
\hline RH57 & -do- & -do- & $8.9 \pm 1.8$ \\
\hline RH61 & -do- & Irregular & $7.4 \pm 1.2$ \\
\hline RH53 & -do- & -do- & \\
\hline
\end{tabular}

* Significantly less than that of the parental strain RH41 $(p<0.05)$

$\checkmark$ No significant differences from uninoculated control

${ }^{\text {a }}$ Each mean dry shoot weight value is a mean of dry shoot weights of eight plants. 
Similar results for the anthranilate synthase mutants of $S$. meliloti were obtained by Barsomian et al. (33) who suggested that anthranilic acid may act as in planta siderophore, promoting iron uptake which is essential for heme and nitrogenase synthesis and thereby the maturation of bacteroids. Normal nitrogen fixation in the mutants blocked at the last step of tryptophan biosynthesis indicates that tryptophan is available to bacteroids from the plant host, i.e. the clover plant supplies tryptophan. Prasad et al. (34) also found that alfalfa plants supplies tryptophan to $S$. meliloti bacteroids. Normal supply of anthranilic acid and at least one more intermediate (unspecified) of tryptophan biosynthesis in these bacteria appears to be necessary for normal symbiotic activity with clover plants.

Abnormal nodule development and defectivity in nitrogen fixation in the mutants of tyrosine (RH61) and phenylalanine (RH53) of the $R$. leguminosarum bv. trifolii mean that clover plant does not supply requirement to the microsymbiont. Kerppola and Kahn (12) also reported that the tyrosine mutants $S$. meliloti 104A14 induces ineffective nodules on alfalfa plants, whereas Prasad et al. (34) found normal symbiotic activity $\left(\mathrm{Nod}^{+} \mathrm{Fix}^{+}\right)$for a Tn5-induced tyrosine auxotroph of S. meliloti Rmd201. Phenylalanine auxotrophic mutant of $S$. meliloti Rmd201 was found to form nodules on alfalfa plants but the nodules were completely ineffective in nitrogen fixation.

\section{References}

1) Schultze M. and kondorosi A. (1998). Annu. Rev. Genet., 32: 33-57.

2) Irvving H. R.; Boukli N. M.; Kelly M. N. and Broughton W. J. (2000). "Root Hairs, Cell and Molecular Biology". Springer-Verlag, Tokyo, Japan, Pp. 242.

3) Long S. R. (2001). Plant Physiol., 125: 69-72.

4) Randhawa G. S. and Hassani R. (2002). Indian J. Exp. Biol., 40: 755764.

5) Zang H.; D'Aoust F.; Charles T. C.; Driscoll B.; Prithiviraj B. and Smith, D. L. (2002). Crop Scie., 42: 1186-1190.

6) Singh R. K. (2001). Indian J. Exp. Biol., 39: 818-820.

7) McLaughlin W. and Ahmad M. H. (1987). Current Microbiol., 15: 301303. 
8) Skogen-Hagenson M. J. and Atherly A. G. (1983). J. Bacteriol., 156: 937-940.

9) Mishima E.; Hosokawa A.; Imaimi-Anraku H.; Saito K.; Kawaguchi M. and Saeki K. (2008) Plant Cell Physiol., 9: 1093-1104.

10) Dénarié J.; Truchet G. and Bergeron B. (1976). Effects of some mutations on symbiotic properties of Rhizobium. In: Symbiotic nitrogen fixation. Nutman P. S. (ed.), Cambridge: Cambridge University Press, Pp. 47.

11) Fedorov S. N. and Zaretskaya A. N. (1977). Mikrobiologiya, 47: 728732.

12) Kerppola T. K. and Kahn M. K. J. (1988). Gen. Microbiol., 134: 913919.

13) Kim C. H.; Kuykendall L. D.; Shah K. S. and Keister D. L. (1988). Appl. Environ. Microbiol., 54: 423-427.

14) 14. Hom S. S. M.; Uratsu S. L. and Hoang F. (1984). J. Bacteriol., 159: 335-340.

15) de Bruijn F. J.; Rossbach S.; Schneider M.; Ratet P.; Messmer S.; Szeto W. W.; Ausubel F. M. and Schell J. (1989). J. Bacteriol., 171: 16731682.

16) Scherrer A. and Dénarié J. (1971). Plant Physiol., (special vol.), 39-45.

17) Malek W. and Kowalski M. (1977). Acta Microbiol. Pol., 26: 351-359.

18) Hassani R.; Prasad C. K.; Vineetha K. E.; Vij N.; Singh P.; Sud R.; Yadav S. and Randhawa G. S. (2002). Indian J. Exp. Biol., 40:11101120.

19) Forrai T.; Vincze E.; Banfalvi Z.; Kiss G. B.; Randhawa G. S. and Kondorosi A. (1983). J. Bacteriol., 153: 635-643.

20) King N. D.; Hojnacki D. and O’Brian M. R. (2000). Appl. Environ. Microbiol., 66: 5469-5471.

21) Vincent J. M. (1970). "A manual for the Practical Study of the RootNodule Bacteria". I. B. P. Handbook No. Blackwell Scientific Publication Ltd., Oxford, U. S. A, Pp. 164.

22) Khanuja S. P. S. and Kumar S. (1988). Indian J. Exp. Biol., 26: 665667.

23) Swamynatha S. K. and Singh A. (1995). J. Biosci., 20:17-28. 


\section{Dr. Raad Hassani Sultan}

24) Khanuja S. P. S. and Kumar S. J. (1989). Genet., 68: 93-108.

25) Singh A., Ram J., Sikka V. K. and Kumar S. (1984). Indian J. Exp. Biol., 22: 239-247.

26) Fahraeus G. J. (1957). Gen. Microbiol., 16: 374-381.

27) Davis R. W.; Botstein D. and Roth J. R. (1980). “Advanced Bacterial Genetics, A manual for Genetic Engineering". Cold Spring Harbor, N. Y. Cold Spring Harbor Laboratory, U. S. A, Pp. 209.

28) Snell F. D. and Snell C. T. (1967). "Colorimetric Methods of Analysis Including photometric Methods". D. Van Nostrand Company Inc., New Jersey, U. S. A.

29) Yanofsky C. and Smith O. H. (1962). Methods Enzymol., 5: 794-801.

30) Wang C.; Sheng X.; Equi R. C.; Trainers M. A.; Charles T. C. and Sobral B. W. S. (2007). J. Bateriol., 184: 9050-9056.

31) Somasegaran P. and Hoben H. J. Handbook for Rhizobia. In: Methods in Legume-Rhizobium Technology. Springer-Verlag. New York. Pp. 402-408.

32) Moat A. G. and Foster J. W. (1995). Amino acids, Purines and pyrimidines, In: Microbial Physiology. A John Wiley and Sons, INC, Publication, U.S.A., Pp. 486.

33) Barsomian G. D.; Urzainqui A.; Lohman K. and Walker G. C. (1992). J. Bacteriol., 174-184.

34) Prasad C. K., Vineetha K. E., Hassani R. and Randhawa G. S. (2000). Indian J. Exp. Biol., 38: 1041-1049. 